\title{
AVALIAÇÃO DE CLIMA ORGANIZACIONAL UTILIZANDO UMA ABORDAGEM MULTICRITÉRIO DE APOIO À DECISÃO
}

\author{
Gerson Ulbricht \\ IFSC - CEP 89252-020 - Jaraguá do Sul - SC. \\ e-mail: gerson.ulbricht@ifsc.edu.br \\ Neida M. Patias Volpi \\ UFPR - CEP 81531-990 - Curitiba - PR. \\ e-mail: neida@ufpr.br \\ Ariene Sampaio Souza Farias Ulbricht \\ IFSC - CEP 89252-020 - Jaraguá do Sul - SC. \\ e-mail: arieneu@ibest.com.br \\ Sander Joner \\ UFPR - CEP 81531-990 - Curitiba - PR. \\ e-mail: sander.joner@ifsc.edu.br.
}

\section{RESUMO}

Este artigo mostra um modelo para avaliação de clima organizacional que pode ser aplicado em diferentes tipos de instituições. Parte-se da aplicação de questionários que são compostos de critérios avaliativos e que em uma primeira fase são respondidos pelos entrevistados. Após essa fase, faz-se a transposição da tabela de dados onde cada alternativa torna-se um critério de avaliação e vice-versa. Esse procedimento permite classificar quais os critérios de avaliação são de destaque bem como quais são mais deficientes de modo a contribuir para uma análise do cenário e possíveis providências. Devido à característica dos dados, é dado ênfase à metodologia multicritério PROMETHEE II - Preference Ranking Organization METHod for Enrichment Evaluations a qual demonstrou ótimos resultados neste estudo.

PALAVRAS CHAVE: Clima Organizacional, PROMETHEE II. Área principal: Métodos de Apoio à Decisão Multicritério.

\begin{abstract}
This paper shows a model for assessing organizational climate that can be applied in different types of institutions. Part from the questionnaires that are composed of evaluative criteria and that in the first phase are answered by respondents. After this phase, it is the transposition of data where each alternative becomes an evaluation criterion and vice versa table. This procedure allows you to sort the criteria for evaluation are highlighted and which are poorer in order to contribute to a scenario analysis and possible action. Due to the characteristic of the data is given emphasis to multicriteria method PROMETHEE II - Preference Ranking Organization Method for Enrichment Evaluations which has shown great results in this study.
\end{abstract}

KEYWORDS: Organizational Climate, PROMETHEE II.

Main area: Methodologies Multicriteria Decision Support. 


\section{INTRODUÇÃO}

Considerando as estruturas organizacionais cada vez mais complexas, o processo de tomada de decisão de uma empresa necessita, cada vez mais, ser rápido e eficiente a fim de promover uma boa posição estratégica. As incertezas de mercado e de produção levam as empresas a investir em flexibilidade na busca por maior robustez, Branco (2010). Um fator de grande importância para o desempenho no ambiente empresarial é satisfação do colaborador com o ambiente de trabalho.

Diversas técnicas de avaliação sobre a satisfação de colaboradores tem sido empregadas nos ambientes de trabalho. Há problemas de tomada de decisão onde diversos pontos de vista, geralmente conflitantes entre si, devem ser levados em consideração. Deste modo, muitas vezes não existe uma solução ótima que atenda a todos os critérios simultaneamente e sendo assim é necessário encontrar soluções que melhor atendam a esses critérios (Ensslin, 2001).

Os métodos de ajuda na decisão multicritério - MCDA (Multiple Criteria DecisionAid), oferecem ferramentas que contribuem na tomada de decisão de modo que consideram mais de um aspecto e, portanto, avaliam as ações segundo um conjunto de critérios (Almeida, 2013). Cada um dos critérios a serem avaliados é representado por uma função matemática responsável onde esta avalia a performance baseado no peso atribuído. Em problemas multicritério, o objetivo é o de otimizar todas essas funções simultaneamente, o que normalmente não é possível (Ulbricht, 2010).

As metodologias multicritério são aplicadas a problemas de escolha, classificação ou ordenação das alternativas, onde se tem um número conhecido de critérios que se deseja otimizar. Em aplicações práticas geralmente, é impossível se chegar uma solução ótima, ou seja, a melhor em relação a todos os critérios. Busca-se dessa maneira, soluções que atendam da melhor forma possível aos critérios estabelecidos para as alternativas consideradas.

Neste artigo propõe-se o uso de técnicas de avaliação multicritério que possam contribuir para que se tenha uma visão mais estratégica sobre quesitos avaliados no que se refere ao clima organizacional em ambientes empresariais.

\section{FUNDAMENTAÇÃO TEÓRICA}

No cenário dos dias atuais é necessário para o sucesso das organizações estabelecer um ambiente agradável de trabalho visando o benefício coletivo, e facilitando o convívio dos colaboradores. Uma das maneiras de tentar estabelecer um bom clima organizacional,é identificar fatores deficientes e buscar trabalhar para melhorá-los.

De acordo com Campello e Oliveira (2008) a palavra clima origina-se do grego klimae que significa tendência ou inclinação. O clima é algo que não se pode ver ou tocar, mas ele é facilmente percebido dentro de uma organização através do comportamento dos seus colaboradores. Estudos de clima são particularmente úteis, porque fornecem um diagnóstico geral da empresa, bem como indicações de áreas carentes de uma atenção especial. Não basta "sentir" que o clima está mau, é preciso identificar onde, porque e como agir para melhorá-lo. (Pereira, 2013). O clima organizacional é favorável quando proporciona satisfação das necessidades pessoais dos participantes, produzindo elevação do moral interno. É desfavorável quando proporciona frustração daquelas necessidades Chiavenato (2003).

A pesquisa de clima é de grande importância, pois procura medir os níveis de satisfação dos colaboradores, seus aspectos positivos e negativos, permitindo assim a oportunidade de melhorias do clima. Os estudos sobre clima organizacional são úteis por proverem um diagnóstico geral para a organização, indicando as áreas mais deficitárias. (Torres e Oliveira, 2007). 
De modo a procurar melhor avaliar os níveis de resposta encontrados em questionários aplicados, faz-se necessário a utilização de ferramentas de análise de dados. Neste trabalho foi proposto a utilização de metodologias multicritério de apoio à decisão.

Em problemas multicritério a qualidade da decisão além de se basear na qualidade dos modelos matemáticos desenvolvidos, se baseia também nas preferências do decisor e no conhecimento que estarão disponíveis no instante da tomada de decisões (Araújo, 2009). O decisor é uma pessoa ou grupo de pessoas responsável por definir os critérios de avaliação, a importância de cada um dos critérios (escolha de pesos), limiares até onde a diferença entre duas avaliações aplicadas é significativa ou não, entre outros fatores.

A importância do decisor é de fundamental importância e o estudo sobre determinada situação e assim sendo, pode envolver pesquisas, reuniões, discussões, opiniões ou convicções de modo a estabelecer os valores representativos utilizados no sistema. Isso geralmente traz dificuldades, pois em grande parte das vezes é difícil a escolha da importância de cada critério de modo a estabelecer pesos para sua utilização, bem como na definição das diferenças significativas entre valores atribuídos às alternativas (Ulbricht, 2010). Neste artigo, devido à característica dos dados, é dado ênfase à metodologia multicritério PROMETHEE II Preference Ranking Organization METHod for Enrichment Evaluations (Brans e Vincke, 1985) a qual será aplicada a um processo de avaliação de clima organizacional.

\section{METODOLOGIA MULTICRITÉRIO PROMETHEE I E II}

As metodologias multicritério PROMETHEE, consideram critérios a serem maximizados ou minimizados de forma não compensatória (Araz, 2006), ou seja, um baixo valor em um dos critérios não é compensado por um alto valor em outro critério assim como ocorre, por exemplo, em médias aritméticas ou ponderadas.

Um problema multicritério com número $k$ de critérios e um conjunto $A$ de alternativas, pode ser representado por (Gonçalves, 2001):

$$
\operatorname{Maximizar}\left\{f_{1}(a), \ldots, f_{k}(a) / a \square A\right\},
$$

onde: $A=\left\{a_{1}, a_{2}, \ldots, a_{n}\right\}$ é o conjunto de alternativas e $f_{j}, j=1,2, \ldots, k$, são os critérios de decisão a serem considerados.

Enquanto a metodologia PROMETHEE I gera uma ordenação parcial das alternativas, a metodologia multicritério PROMETHEE II gera uma ordenação completa permitindo compará-las em sua totalidade.

As alternativas são comparadas mediante uma função de preferência (Brans e Mareschal, 1999), a qual é uma representação matemática da forma como se tomaria a decisão sobre a preferência por uma alternativa quando comparada a outra.

São encontradas na literatura 6 tipos de funções de preferência utilizadas na família de metodologias multicritério PROMETHEE (Brans e Mareschal, 1999). Podem ser utilizados outros tipos de funções, mas de modo geral, estas funções tornam-se suficientes.

Em todas as funções considera-se $d_{j}(a, b)=f_{j}(a) \square f_{j}(b)$, para critérios a serem maximizados, e $d_{j}(a, b)=f_{j}(b) \square f_{j}(a)$ para critérios a serem minimizados.

Para a implementação das metodologias PROMETHEE I e PROMETHEE II, os dados devem ser organizados numa matriz onde as linhas representam as alternativas às quais se deseja classificar e as colunas representam os critérios de classificação.

A matriz $M$ representa as avaliações dos $k$ critérios onde $A$ é o conjunto das $m$ alternativas consideradas. Assim, $f_{j}\left(a_{i}\right)$, corresponde à avaliação da ação $a_{i}$ segundo o critério $j,(j=1,2, \ldots, k)$ a ser maximizado ou minimizado. 


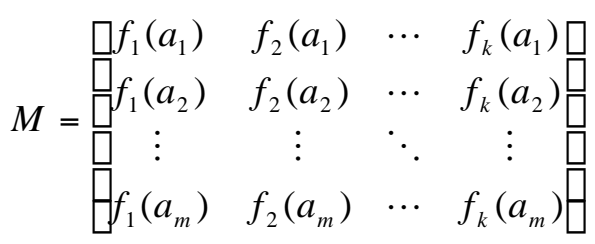

O método consiste em fazer uma comparação entre pares de alternativas pertencentes a essa matriz, onde se deve especificar uma função de preferência $P_{j}$ para cada critério. As funções de preferência são apresentadas no quadro 1.

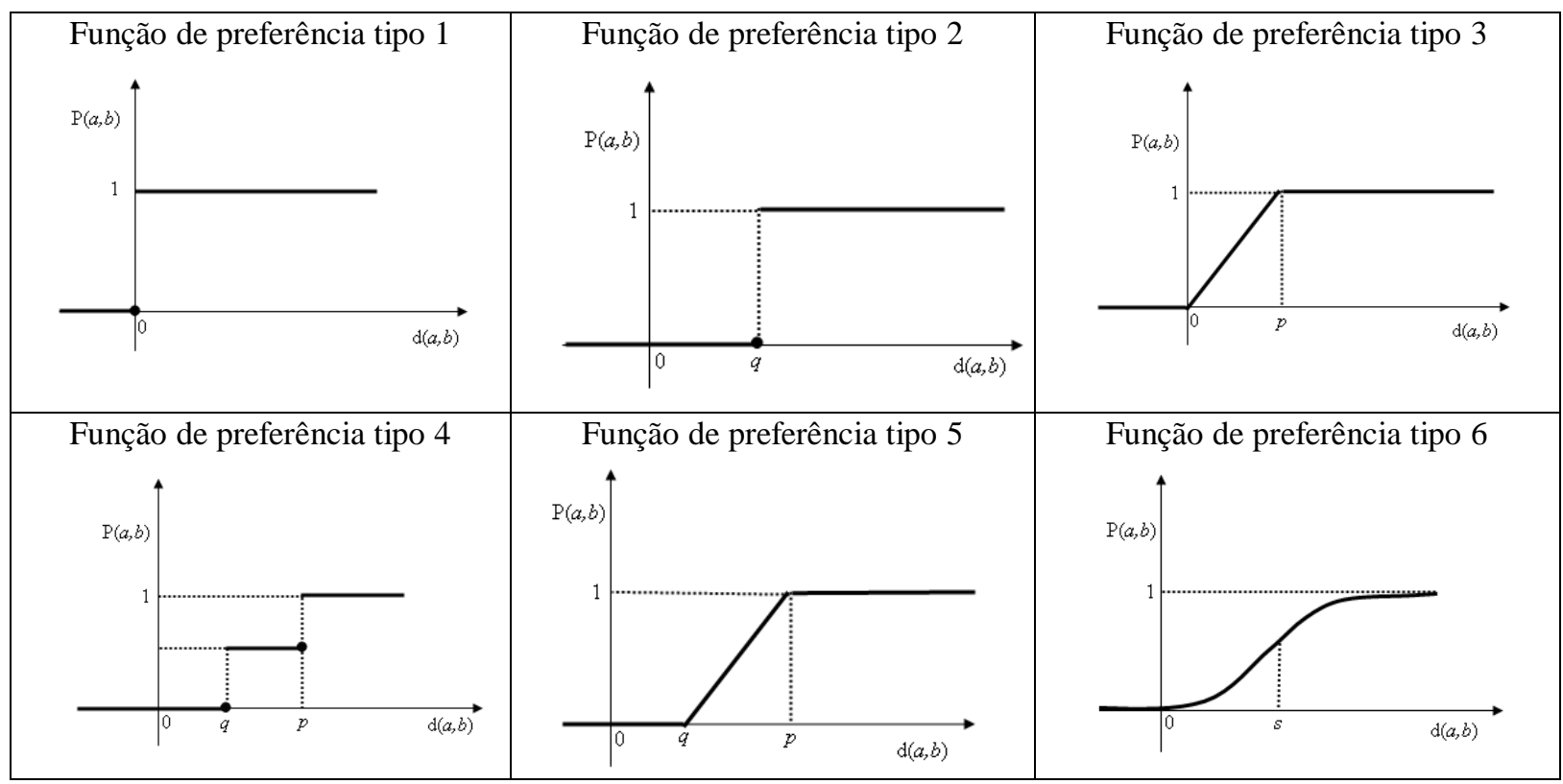

Quadro 1:Funções de preferência.

A função de preferência $P_{j}$ representa a intensidade de preferência da alternativa $a$ em relação à alternativa $b$ onde $0 \leq P_{j} \leq 1$. A interpretação atribuída aos valores de preferência é representada a seguir:

$P_{j}(a, b)=0$ quando não há preferência de $a$ em relação a $b$, referente ao critério $j$;

$P_{j}(a, b) \sim 0$ quando há preferência fraca de $a$ em relação a $b$, referente ao critério $j$;

$P_{j}(a, b) \sim 1$ quando há preferência forte de $a$ em relação a $b$, referente ao critério $j$;

$P_{j}(a, b)=1$ quando há preferência estrita de $a$ em relação a $b$, referente ao critério $j$.

A função de preferência $P_{j}(a, b)$ é o resultado de uma diferença entre duas avaliações:

$$
P_{j}(a, b)=P_{j}\left[d_{j}(a, b)\right] \text { tal que } a, b \sqcup A
$$

Cabe ao tomador de decisão, baseado em sua experiência, definir uma função de preferência $P_{j}$ e um peso $w_{j}$ para cada critério do modelo. Pode ocorrer inclusive de o especialista julgar todos os critérios como tendo o mesmo grau de importância, atribuindo assim pesos iguais a todos os critérios. Os pesos $w_{j}$ atribuídos a cada critério devem ser valores compreendidos entre 0 e 1 onde:

$$
\prod_{j=1}^{k} w_{j}=1
$$

O índice de preferência ponderada $\pi$ deve ser calculado para todos os pares de alternativas sendo que este indica o grau de preferência de uma alternativa $a$ em relação a uma alternativa $b$, já considerando todas as funções de preferência:

$$
\square(a, b)=\prod_{j=1}^{k} w_{j} \cdot P_{j}(a, b)
$$


Deve-se em seguida calcular os índices de preferência positivo e negativo. O índice de preferência positivo, $\square^{+}(a)$ representa a média de índices de preferência de uma alternativa $a$ sobre todas as outras alternativas do conjunto A. Quanto maior $\square^{+}(a)$, melhor a alternativa. $\mathrm{O}$ índice de preferência positivo é dado por:

$$
\square^{+}(a)=\frac{1}{m \square 1} \square \square(a, x)
$$

$$
\square^{+}: A \circledast[0,1]
$$

O índice de preferência negativo $\nabla(a)$ representa a média de preferência de todas as alternativas sobre a alternativa $a$. Quanto menor $\nabla^{\nexists}(a)$, melhor a alternativa.

$$
\begin{aligned}
& \nabla(a)=\frac{1}{m \square 1} \bigsqcup_{x \square A} \square(x, a) \\
& \nabla^{]}: A{ }^{\circledR}[0,1]
\end{aligned}
$$

A ordenação das alternativas é obtida a partir dos índices de preferência $\square^{+}(a)$ e $\nabla(a)$ apresentados em (1) e (2). Baseado nos índices $\square^{+}(a)$ e $\square^{\top}(a)$ é possível fazer a classificação parcial das alternativas, o que é chamado de metodologia multicritério PROMETHEE I (Brans e Mareschal, 1999) onde:

A alternativa $a$ é preferível à alternativa $b(a P b)$ se: $\square^{+}(a)>\square^{+}(b)$ e $\square^{\top}(a)<\square^{\top}(b)$ ou $\square^{+}(a)=\square^{+}(b)$ e $\square^{\square}(a)<\square^{\square}(b)$ ou $\square^{+}(a)>\square^{+}(b)$ e $\square(a)=\square^{\square}(b)$.

A alternativa $a$ é indiferente à alternativa $b(a I b)$ se: $\square^{+}(a)=\square^{+}(b)$ e $\square^{\square}(a)=\square^{\square}(b)$.

A alternativa $a$ é incomparável à alternativa $b(a R b)$ em casos diferentes.

Normalmente poucas classificações são feitas. A maior parte das situações resulta em incomparabilidade. Empregando um novo índice, o qual usa dos índices de preferência positivo e negativo, obtém-se uma ordenação completa das alternativas, o que é chamado de metodologia multicritério PROMETHE II (Brans e Mareschal, 1999). Essa classificação é obtida através da diferença entre os índices de preferência positivo e negativo, onde deste modo, todas as alternativas são comparáveis:

Assim:

$$
\square(a)=\square^{+}(a) \square \nabla^{\square}(a)
$$

i) A alternativa $a$ é preferível à alternativa $b(a P b)$ se $\triangle(a)>\triangle(b)$

ii) A alternativa $a$ é indiferente à alternativa $b(a I b)$ se $\square(a)=\square(b)$

\section{METODOLOGIA}

Neste tópico é mostrada uma metodologia para aplicação da metodologia Metodologia Multicritério PROMETHEE II em um processo de avaliação de clima organizacional que pode ser aplicado em empresas ou outra instituição onde se tenha como objetivo a busca por melhorias no ambiente de trabalho, avaliando assim o relacionamento em equipe, a postura dos integrantes e a relação diferentes níveis hierárquicos na organização.

A metodologia utilizada para avaliação aqui proposta consiste de duas fases sendo que na primeira, cada colaborador de uma instituição é encarregado de responder um questionário contendo várias perguntas referentes aos diversos aspectos que se deseja avaliar. Estas perguntas podem ser redigidas considerando diversos assuntos.

Sendo assim, parte-se para uma segunda fase onde se propõe sobre um ponto de vista matemático, transpor a matriz de avaliações gerada na fase 1, transformando assim, os 
critérios de avaliação anteriormente definidos, em alternativas e as alternativas em critérios de avaliação. Portanto, cada colaborador que seria uma alternativa, passa a ser um critério de avaliação e cada critério, passa a ser uma alternativa, conforme ilustrado na figura 1.

\begin{tabular}{|c|c|c|c|c|}
\hline & 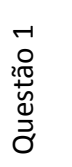 & 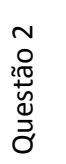 & $\ldots$ & 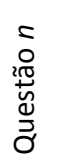 \\
\hline Colaborador 1 & $a_{11}$ & $a_{12}$ & $\ldots$ & $a_{1 n}$ \\
\hline Colaborador 2 & $a_{21}$ & $a_{22}$ & $\cdots$ & $a_{2 n}$ \\
\hline Colaborador 3 & $a_{31}$ & $a_{32}$ & $\cdots$ & $a_{3 n}$ \\
\hline$\vdots$ & $\vdots$ & $\vdots$ & $\vdots$ & $\vdots$ \\
\hline Colaborador $m$ & $a_{m 1}$ & $a_{m 2}$ & $\ldots$ & $a_{m n}$ \\
\hline
\end{tabular}

Alternativas: colaboradores

Critérios de avaliação: questões

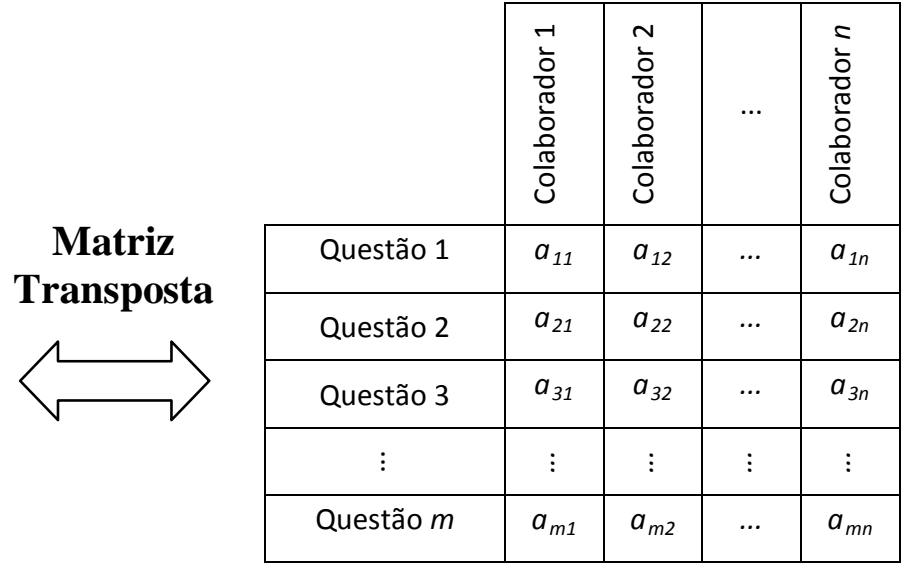

Alternativas: questões

Critérios de avaliação: colaboradores

Figura 1: Transposição da Matriz de Avaliações.

Assim pode-se considerar nessa segunda fase, todos os critérios com o mesmo peso para o modelo matemático, pois cada um destes representa a opinião de um colaborador da instituição e todas as opiniões devem ter o mesmo nível de importância para a construção de uma análise coletiva no ambiente corporativo.

\section{APLICAÇÃO EM UM PROCESSO DE AVALIAÇÃO ORGANIZACIONAL}

Apresenta-se a seguir, uma breve aplicação da metodologia proposta em um processo de avaliação em uma instituição. A aplicação ocorreu por meio de um questionário com 10 questões o qual foi aplicado para 15 colaboradores. A primeira etapa, refere-se à coleta das informações a partir dos questionários aplicados onde cada pergunta é um critério de avaliação e cada colaborador é uma alternativa.

Para alternativas de resposta nas perguntas, pode-se usar uma escala de afetos positiva e negativa tipo Likert, a qual consiste em um tipo de escala de resposta psicométrica usada habitualmente em questionários (Zanon, 2013). Nesta escala, a cada alternativa de resposta atribui-se um valor numérico. O formato típico de um item Likert é: 1:Não concordo totalmente; 2:Não concordo parcialmente; 3:Indiferente; 4:Concordo parcialmente; 5:Concordo totalmente.

Outro exemplo de alternativa de resposta é solicitar para que o respondente avalie cada critério em uma escala de 0 a 10 conforme o grau de satisfação.

Na presente aplicação foram utilizadas 10 perguntas, as quais são apresentados no quadro 1 , onde os respondentes avaliaram cada item com notas de 0 a 10 conforme o grau crescente de satisfação. 


\begin{tabular}{|c|l|}
\hline $\mathbf{N}^{\mathbf{o}}$ Questão & \multicolumn{1}{c|}{ Critério Avaliativo } \\
\hline 1 & Satisfação com o ambiente físico de trabalho \\
\hline 2 & Valorização capacidade profissional \\
\hline 3 & Confiança em colegas de trabalho \\
\hline 4 & Comunicação entre colaborador e supervisor direto \\
\hline 5 & Sintonia da equipe de trabalho \\
\hline 6 & Satisfação com a remuneração \\
\hline 7 & Satisfação com a segurança no ambiente de trabalho \\
\hline 8 & Uso do diálogo para resolver problemas de trabalho \\
\hline 9 & Equipamentos necessários para realizar as funções no ambiente de trabalho \\
\hline 10 & Valorização pela instituição \\
\hline
\end{tabular}

Quadro 1: Questões que compõe o questionário avaliativo (critérios avaliativos).

Considerando a Metodologia Multicritério PROMETHEE II, cada colaborador que respondeu a um questionário seria uma alternativa a qual poderia ser classificada através de uma lista de preferência (ranking). A proposta deste trabalho não é esse tipo de classificação pois esse procedimento poderia causar tensões indesejadas dentro da organização. O quadro 2 mostra as avaliações realizadas por cada colaborador referente às 10 questões (critérios de avaliação).

\begin{tabular}{|c|c|c|c|c|c|c|c|c|c|c|}
\hline Critério & 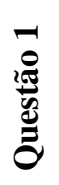 & 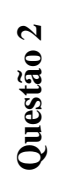 & 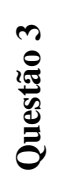 & 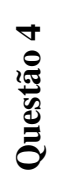 & 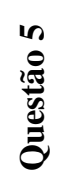 & 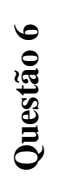 & 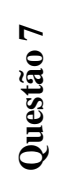 & 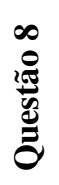 & 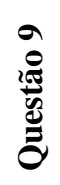 & 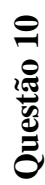 \\
\hline Colaborador 1 & 7 & 8 & 9 & 4 & 4 & 9 & 9 & 7 & 9 & 9 \\
\hline Colaborador 2 & 8 & 8 & 10 & 7 & 5 & 2 & 8 & 7 & 9 & 9 \\
\hline Colaborador 3 & 6 & 9 & 9 & 7 & 6 & 5 & 8 & 6 & 9 & 9 \\
\hline Colaborador 4 & 6 & 7 & 9 & 6 & 7 & 7 & 8 & 7 & 9 & 9 \\
\hline Colaborador 5 & 9 & 7 & 9 & 8 & 7 & 3 & 8 & 7 & 9 & 8 \\
\hline Colaborador 6 & 7 & 8 & 10 & 5 & 7 & 6 & 8 & 7 & 9 & 8 \\
\hline Colaborador 7 & 6 & 8 & 9 & 5 & 7 & 10 & 9 & 7 & 9 & 7 \\
\hline Colaborador 8 & 9 & 7 & 8 & 6 & 5 & 5 & 8 & 7 & 9 & 7 \\
\hline Colaborador 9 & 7 & 8 & 9 & 7 & 10 & 7 & 8 & 7 & 9 & 9 \\
\hline Colaborador 10 & 7 & 9 & 8 & 7 & 7 & 7 & 8 & 7 & 9 & 8 \\
\hline Colaborador 11 & 8 & 9 & 9 & 6 & 8 & 3 & 8 & 7 & 9 & 9 \\
\hline Colaborador 12 & 7 & 10 & 9 & 6 & 6 & 5 & 9 & 7 & 9 & 8 \\
\hline Colaborador 13 & 6 & 9 & 9 & 6 & 7 & 7 & 8 & 7 & 9 & 9 \\
\hline Colaborador 14 & 9 & 8 & 7 & 6 & 5 & 6 & 8 & 7 & 9 & 8 \\
\hline Colaborador 15 & 7 & 7 & 7 & 6 & 5 & 2 & 7 & 7 & 9 & 8 \\
\hline
\end{tabular}

Quadro 2: Matriz de Avaliações.

Os dados do quadro 2 apresentado anteriormente, representam a percepção dos colaboradores em relação aos critérios (questões) avaliados. A partir dos questionários já coletados, parte-se para a segunda fase, onde se tem por objetivo classificar as questões avaliadas de modo a verificar quais características destacam-se positivamente bem como quais destas precisam se melhor trabalhadas na instituição. 


\begin{tabular}{|c|c|c|c|c|c|c|c|c|c|c|c|c|c|c|c|}
\hline Alterna & 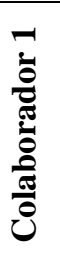 & 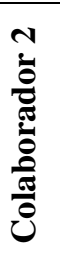 & 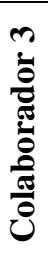 & 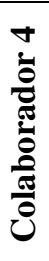 & 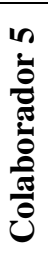 & 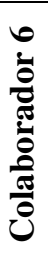 & 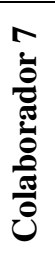 & 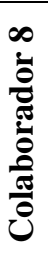 & $\begin{array}{l}0 \\
\vdots \\
\frac{0}{0} \\
\frac{\pi}{0} \\
\frac{0}{0} \\
\frac{\pi}{0} \\
0\end{array}$ & 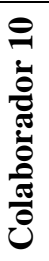 & 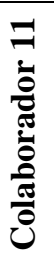 & 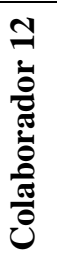 & 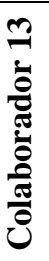 & 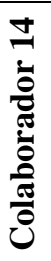 & 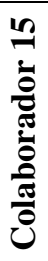 \\
\hline Questão 1 & 7 & 8 & 6 & 6 & 9 & 7 & 6 & 9 & 7 & 7 & 8 & 7 & 6 & 9 & 7 \\
\hline Questão 2 & 8 & 8 & 9 & 7 & 7 & 8 & 8 & 7 & 8 & 9 & 9 & 10 & 9 & 8 & 7 \\
\hline Questão 3 & 9 & 10 & 9 & 9 & 9 & 10 & 9 & 8 & 9 & 8 & 9 & 9 & 9 & 7 & 7 \\
\hline Questão 4 & 4 & 7 & 7 & 6 & 8 & 5 & 5 & 6 & 7 & 7 & 6 & 6 & 6 & 6 & 6 \\
\hline Questão 5 & 4 & 5 & 6 & 7 & 7 & 7 & 7 & 5 & 10 & 7 & 8 & 6 & 7 & 5 & 5 \\
\hline Questão 6 & 9 & 2 & 5 & 7 & 3 & 6 & 10 & 5 & 7 & 7 & 3 & 5 & 7 & 6 & 2 \\
\hline Questão 7 & 9 & 8 & 8 & 8 & 8 & 8 & 9 & 8 & 8 & 8 & 8 & 9 & 8 & 8 & 7 \\
\hline Questão 8 & 7 & 7 & 6 & 7 & 7 & 7 & 7 & 7 & 7 & 7 & 7 & 7 & 7 & 7 & 7 \\
\hline Questão 9 & 9 & 9 & 9 & 9 & 9 & 9 & 9 & 9 & 9 & 9 & 9 & 9 & 9 & 9 & 9 \\
\hline Questão 10 & 9 & 9 & 9 & 9 & 8 & 8 & 7 & 7 & 9 & 8 & 9 & 8 & 9 & 8 & 8 \\
\hline
\end{tabular}

Quadro 3: Matriz de Avaliações Transposta.

A partir dos dados apresentados no quadro 3, é aplicada a metodologia PROMETHEE II. Sendo os critérios neste caso então, formados pelas opiniões dos $k$ colaboradores da organização, e considerando que todas estes possuem o mesmo grau de importância no que se refere às suas opiniões, foram adotados pesos $w$ bem como limiares e indiferença $q$ e preferência estrita $p$, iguais para todos os critérios avaliados. Assim sendo, o valor do peso adotado para cada critério foi $w_{j}=\frac{1}{k}$, sendo $k$ o número de colaboradores que formam nesse caso, os critérios. Considerou-se um limiar de indiferença $q=1$, o limiar de preferência estrita $p=3$, bem como a função de preferência tipo 5 mostrada no quadro 1 desse trabalho, para todos os critérios.

Como a escala de notas está situada em um intervalo de 1 a 10 pontos, optou-se pelos limiares definidos acima, considerando-se uma variação de 1 ponto, indiferente, enquanto que a variação de 3 pontos, de total preferência. A função de preferência tipo 5 admite que entre os limiares $q$ e $p$, a preferência vai aumentando linearmente. Esses valores $q$ e $p$ são atribuídos pelo decisor e devem ser baseados na sua experiência prévia ou em comum acordo com os envolvidos no processo.

O quadro 4 apresenta os resultados da aplicação da metodologia PROMETHEE II nos 10 quesitos avaliados pelos colaboradores. Neste quadro os critérios foram organizados conforme ordem de importância a serem resolvidos, partindo dos que receberam piores avaliações para os que receberam melhores avaliações.

\begin{tabular}{|c|c|c|c|}
\hline Questão & $\boldsymbol{\varphi}^{+}$ & $\boldsymbol{\varphi}^{-}$ & $\boldsymbol{\varphi}$ \\
\hline Questão 6 & 0,0630 & 0,4963 & $\mathbf{- 0 , 4 3 3 3}$ \\
\hline Questão 4 & 0,0370 & 0,4037 & $\mathbf{- 0 , 3 6 6 7}$ \\
\hline Questão 5 & 0,0778 & 0,3704 & $\mathbf{- 0 , 2 9 2 6}$ \\
\hline Questão 8 & 0,0741 & 0,2111 & $\mathbf{- 0 , 1 3 7 0}$ \\
\hline Questão 1 & 0,1407 & 0,2074 & $\mathbf{- 0 , 0 6 6 7}$ \\
\hline Questão 7 & 0,2000 & 0,0148 & $\mathbf{0 , 1 8 5 2}$ \\
\hline Questão 2 & 0,2444 & 0,0444 & $\mathbf{0 , 2 0 0 0}$ \\
\hline Questão 10 & 0,2444 & 0,0333 & $\mathbf{0 , 2 1 1 1}$ \\
\hline Questão 3 & 0,3259 & 0,0148 & $\mathbf{0 , 3 1 1 1}$ \\
\hline Questão 9 & 0,3889 & 0,0000 & $\mathbf{0 , 3 8 8 9}$ \\
\hline
\end{tabular}

Quadro 4: Classificação das Alternativas pelo PROMETHEE II. 
Analisando os resultados apresentados no quadro 4, é possível perceber que os critérios 9 (Equipamentos necessários para realizar as funções no ambiente de trabalho) e 3 (Confiança em colegas de trabalho ) são os mais bem atendidos. Observa-se, porém, fragilidades quanto ao critério 6 (Satisfação com a remuneração) e 4 (Comunicação entre colaborador e supervisor direto), sendo deste modo necessário ser implementadas ações que busquem esclarecer melhorar estes aspectos.

Diversas conclusões sobre os demais itens podem ser tomadas conforme observa-se a ordem de importância mostrada na coluna $\varphi$ do quadro 4.

\section{CONCLUSÕES}

Neste trabalho foi apresentada uma metodologia de avaliação de clima organizacional que pode ser aplicada em qualquer instituição onde deseja-se traçar um perfil do relacionamento e satisfação de seus colaboradores. A análise dos critérios de avaliação através da metodologia multicritério PROMETHEE II apresenta como vantagem a possibilidade da alteração dos pesos atribuídos aos critérios, das funções de preferência e dos limiares de indiferença e preferência estrita conforme interesse do decisor, gerando nova ordem classificatória o que é de extremo interesse em um processo de avaliação.

Mostra-se que com a aplicação da metodologia PROMETHEE II é possível estabelecer prioridades que devem ser trabalhadas no ambiente empresarial de forma a buscar melhorias que possam contribuir para o clima organizacional.

Um dos objetivos da temática desse trabalho foi demonstrar que tão importante quanto a conscientização e transparência em um processo de avaliação é o tratamento adequado dos dados obtidos. Esse tipo de tratamento utilizando técnicas matemáticas apresentadas através da aplicação em um caso específico, serve como exemplo de modelo para análise de dados e pode ser adaptado para utilização em outros processos de avaliação nas organizações. Neste modelo, busca-se identificar através da análise dos critérios avaliativos, aspectos relevantes, fornecendo assim uma ferramenta alternativa para a gestão institucional no auxílio para a tomada de decisão.

\section{REFERÊNCIAS}

[1] Almeida, A. T. Processo de Decisão nas Organizações: Construindo Modelos de Decisão Multicritério. São Paulo: Editora Atlas, 2013.

[2] Araújo, A. G.; Almeida, A. T. Apoio à decisão na seleção de investimentos em petróleo e gás: uma aplicação utilizando o método PROMETHEE. Gest. Prod. vol.16 no.4 São Carlos Oct./Dec. 2009

[3] Araz, Ceyhun. Ozkarahan, Irem. Supplier evaluation and management system for strategic sourcing based on a new multicriteria sorting procedure. International Journal of Production Economics. 106 (2007), 585-606.

[4] Brans, Jean P., Mareschal. Multicriteria Decision Aid: The PROMETHEE GAIA Solution. Pesquisa Operacional, Vol. 19, 06/1999, p. 1-23.

[5] Brans, J. P.; Vincke, P. H. A preference ranking organization method: (The PROMETHEE Method for Multiple Criteria Decision-Making). Management Science, v. 31, p. 647-656, 1985.

[6] Campello, M.L.C.;Oliveira, J.S.G. Clima organizacional no desempenho das empresas, Bauru,2008 
[7] Chiavenato, Idalberto, Introdução à Teoria Geral da Administração. 7. ed. rev. e atual. Rio de Janeiro: Elsevier, 2003. 634 p.

[8] Ensslin, L. Montibeller, G. N. Noronha, S. M.. Apoio à Decisão: Metodologias para Estruturação de Problemas e Avaliação Multicritério de Alternativas. Ed. Insular. Florianópolis - SC, 2001.

[9] Gonçalves, João do Carmo Lopes. Aplicação do Algoritmo PROMETHEE em um problema de Decisão Multiobjetivo. Dissertação de Mestrado. Curitiba: UFPR, 2001.

[10] Pereira, F. A. M.; Oliveira, E.; Teixeira, J. C. M. A Influência do Clima e Cultura Organizacional na Gestão em uma Escola. ISSN 16774280 Vol.14. No. 1(2013)

[11] Sousa, E.L.P. Clima e motivação em uma empresa estatal. Rio de Janeiro, 1982. Disponível em: <http://www.scielo.br/scielo>.

[12] Torres, E. F.; Oliveira, J. A. A influência do clima organizacional no alcance da efetividade organizacional em industrias do setor alimentícios utilizando o modelo ASH. Rio de Janeiro-RJ, 2007. Disponível em: <http://www.anpad.org.br>.

[13] Ulbricht, G. Uma abordagem Multicritério na Análise da Avaliação Docente. Ágora V. 16, n. 1 jan/jul., 2010.

[14] Zanon, C. Et all; Desenvolvimento e validação de uma escala de afetos positivos e negativos. Psico-USF, Bragança Paulista, v. 18, n. 2, p. 193-202, maio/agosto 2013. Disponível em www.scielo.br. 